\title{
Optimal Coordination of Robot Motions with Positioner and Linear Track in a Fiber Placement Workcell
}

\author{
Jiuchun Gao ${ }^{1}$, Anatol Pashkevich ${ }^{1}$, Marco Cicellini ${ }^{1}$ and Stéphane Caro ${ }^{2}$ \\ ${ }^{1}$ Laboratoire des Sciences du Numérique de Nantes (LS2N), Institut Mines-Télécom Atlantique, Nantes, France \\ ${ }^{2}$ Laboratoire des Sciences du Numérique de Nantes (LS2N), Centre National de la Recherche Scientifique, Nantes, France \\ \{jiuchun.gao,anatol.pashkevich, stephane.caro\}@ls2n.fr, marco.cicellini@eleves.ec-nantes.fr
}

Keywords: Redundant Robotic System, Composite Fiber Placement, Optimal Motion Coordination.

Abstract: $\quad$ The paper proposes a methodology for optimal coordination of motions in robotic systems with multiple redundant actuators. In contrast to our previous results dealing with a single redundant axis, the extended technique is proposed allowing the robot, positioner and linear track to be actuated simultaneously in order to reduce the total processing time. The developed technique transforms the original continuous problem into a discrete one where the desired time-optimal motions are presented as a shortest path on the task graph satisfying the problem-specific acceleration and velocity constraints imposed on the joint coordinates. The desired time optimal motions are generated using enhanced dynamic programming algorithm that considers both of these constraints. Two case studies are presented to demonstrate efficiency of the approach and evaluate benefits of simultaneous actuation of all robotic system axes.

\section{INTRODUCTION}

Currently, composite materials have been increasingly used in aerospace and automotive industries because of their good strength-to-weight ratio and durability (Pham et al., 2016, Garoushi, 2018). For fabricating complex composite parts, automated fiber placement technique is widely used (Gay, 2014, Frketic et al., 2017). The relevant technological process can be implemented by using either specifically designed CNC machines or robotic systems. Such machines have no limitations on the component size, but they are expensive and usually require large work-floor areas (GalletHamlyn, 2011). In contrast, the robotic systems are relatively cheap and flexible, allowing changing the product type easily. However, they are usually kinematically redundant because of excessive number of actuated axes that are provided by a 6-dof robot, a 1-dof positioner and a 1-dof linear track. For this reason, in robotic fiber placement the optimal coordination of the manipulator motions with the positioner/track movements is an important issue.

In literature, there are a number of works that deal with the redundancy resolution in robotic systems. Relevant techniques are usually based on the pseudo inverse of the kinematic Jacobian (Flacco and De
Luca, 2015). However, they can be hardly applied to the considered problem because they do not allow generating optimal trajectories satisfying real-life industrial requirements (Kazerounian and Nedungadi, 1988). Alternatively, there are also several techniques based on conversion of the original continuous problem to a discrete one. The simplest one is able to generate time-optimal trajectories for point-to-point motions and was applied to the spot-welding (Gueta et al., 2008, Gueta et al., 2017). A slightly different method was proposed in (Pashkevich et al., 2004) for the laser cutting and arc-welding applications where the motion amplitude for the actuated axes was minimized but the tool speed was assumed to be constant.

For the considered process, where the tool speed variations are allowed in certain degree, a discrete optimization based methodology was proposed in our previous work (Gao et al., 2017). It allows the user to convert the original problem to the combinatorial one taking into account particularities of the fiber placement technology and to generate time-optimal coordinated motions for the robot and positioner. However, the technique was applied to a planar benchmark example only, with a single redundant variable. In this work, an extension of the previous results is proposed allowing dealing with 
the optimal motion coordination for robotic systems with higher degree of redundancy, which arises when the robotic manipulator, the positioner and linear track actuated simultaneously.

\section{ROBOTIC FIBER PLACEMENT PROBLEM}

A typical robotic fiber placement workcell is presented in Figure 1. Here, the workpiece to be covered by the composite fiber reinforcement is manipulated by the positioner, which is able to change its orientation in order to improve accessibility of certain zones by the technological tool. This tool is attached to the robot flange and ensures placement of the fiber reinforcement in desired locations. The robot is installed on a translational linear track allowing adjusting its base location while processing relative large products.

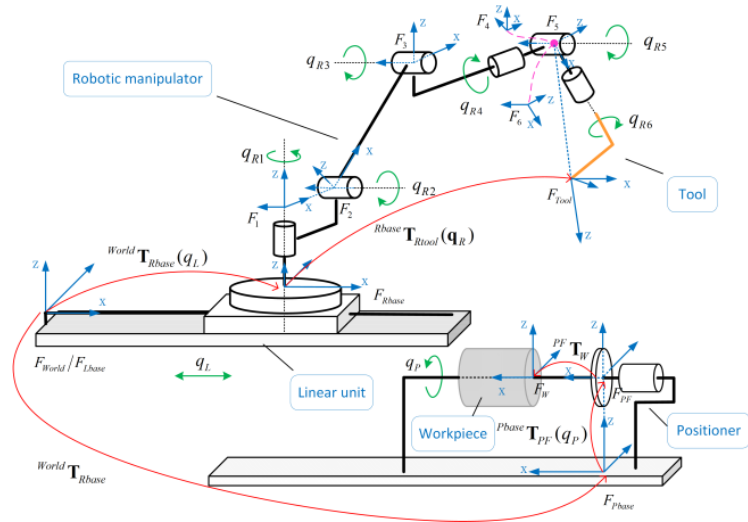

Figure 1: Robotic fiber placement workcell.

In robot-based composite manufacturing, preparation of the manufacturing process includes a number of stages presented in Figure 2, where the motion coordination of all robotic system components is one of the most difficult procedures. Within this process, the desired fiber placement path is generated using a dedicated CAM system and it is presented in a discrete form. Further, the obtained set of task points is transformed into the task graph that describes all possible combinations of the robot, positioner and linear track coordinates. Then, the motion generator produces the optimal trajectories that correspond to the "shortest" path on the task graph. Finally, the obtained motions are converted into the program for the robot control system.

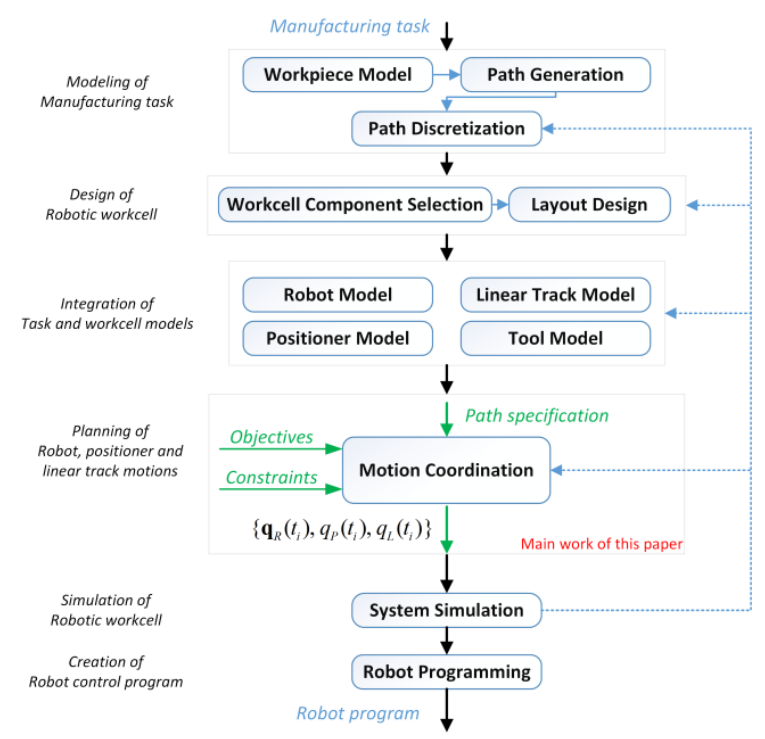

Figure 2: Manufacturing process preparation for robotic fiber placement processes.

\section{SYSTEM KINEMATIC MODEL}

To describe the fiber placement task, let us present it as a sequence of the frames $\left\{F_{\text {task }}^{(i)}, i=1,2, \ldots n\right\}$, in such a way that the $\mathrm{X}$-axis is directed along the path direction and Z-axis is normal to the workpiece surface pointing outside of it. Using these notations, the task locations can be described by a set of $4 \times 4$ homogenous transformation matrices and the considered task is formalized as follows:

$$
{ }^{w} \mathbf{T}_{\text {task }}^{(1)} \rightarrow \ldots{ }^{w} \mathbf{T}_{\text {task }}^{(i)} \rightarrow \ldots{ }^{w} \mathbf{T}_{\text {task }}^{(n)} ; i=1,2, \ldots n
$$

where all vectors of positions and orientations are expressed with respect to the workpiece frame (see superscript "w"). To execute the given fiber placement task, the technological tool must visit the frames defined by (1) as fast as possible.

In any task location, the spatial configurations of the robot, positioner and linear track can be described by the joint coordinates $\mathbf{q}_{R}, q_{p}$ and $q_{L}$. So, the task locations can be expressed using the direct kinematic functions of these components, which are further denoted as $g_{R}\left(\mathbf{q}_{R}\right), g_{p}\left(q_{P}\right)$ and $g_{L}\left(q_{L}\right)$. This allows us to write the kinematic equations describing the given fiber placement task in the following form

$$
\begin{aligned}
& \quad{ }_{\text {World }} \mathbf{T}_{\text {Lbase }} \cdot g_{L}\left(q_{L}^{(i)}\right) \cdot g_{R}\left(\mathbf{q}_{R}^{(i)}\right) \cdot{ }^{\text {Tool }} \mathbf{T}_{\text {task }}= \\
& ={ }^{\text {World }} \mathbf{T}_{\text {Pbase }} \cdot g_{P}\left(q_{P}^{(i)}\right) \cdot{ }^{w} \mathbf{T}_{\text {task }}^{(i)} ; \quad i=1,2, \ldots n
\end{aligned}
$$

where all notations are defined in Figure 1. It is clear that the above equations cannot be solved for $\mathbf{q}_{R}, q_{p}$ 
and $q_{L}$ in unique way because the robotic system is kinematically redundant. On the other side, it gives some freedom for optimizing the coordinated motions of the robotic manipulator with the positioner and linear track movements.

\section{GENERATION OF OPTIMAL COORDINATED MOTIONS}

To present the problem in a formal way, let us define the function $\mathbf{q}_{R}(t), \quad q_{p}(t)$ and $q_{L}(t)$ describing profiles of the robot, positioner and linear track joint coordinates as a function of time $t \in[0, T]$. Additionally, let us introduce a sequence of time instants $\left\{t_{1}, t_{2}, \ldots t_{n}\right\}$ corresponding to the cases when the technological tool visits the task locations (1). So, the considered motion coordination problem can be presented as

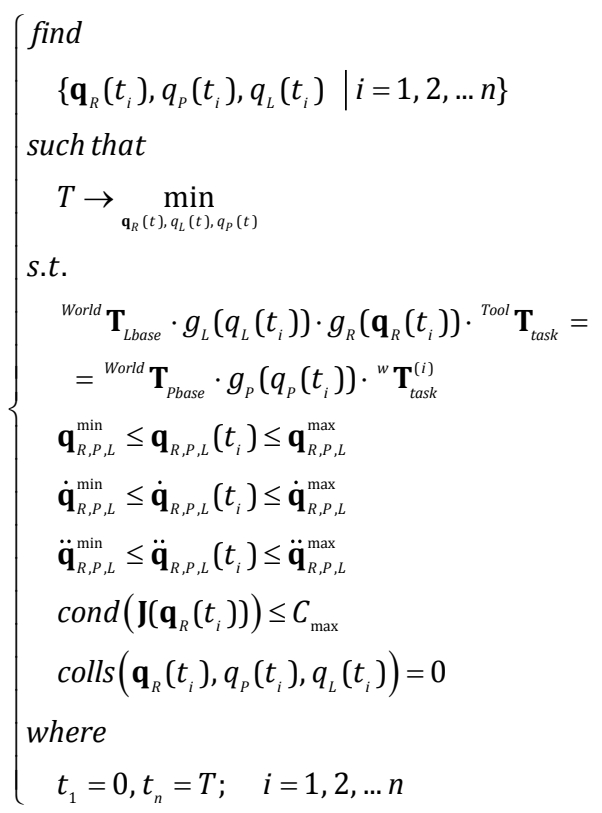

where the main objective is to minimize the total motion time using full capacities of the redundant robotic system, which are limited by the maximum velocity/acceleration values for the actuated joints. Besides, the collision constraints $\operatorname{colls}(\cdot)$ as well as the distance to singularities $\operatorname{cond}(\cdot)$ are also taken into account.

Because of specific constraints, the above presented continuous optimization problem cannot be solved in a straightforward way. For this reason, the considered problem is converted into a discrete form by sampling the redundant variables corresponding to the positioner and the linear track. Then, using ideas proposed in our previous work (Gao et al., 2017) and applying sequentially the direct kinematics of the positioner and linear track as well as the inverse kinematics of the robot, one can get a configuration state for the robotic system in joint space (see Figure 3). This allows generating an extended task graph where all task locations are ordered in time. This graph contains all possible configuration states of the considered robotic system for executing the given fiber placement path, and the desired time-optimal solution of the relevant optimization problem is presented as the shortest path connecting the initial and the final layers. An outline of the task graph generation algorithm is presented in Appendix A.

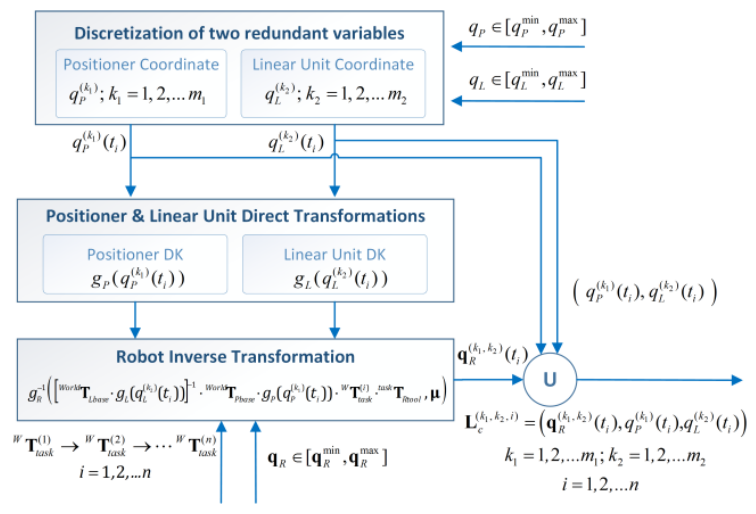

Figure 3: Transformation of the original continuous problem into discrete form.

The structure of this 3D graph is presented in Figure 4 where the nodes $\left\{\mathbf{L}_{\text {task }}^{\left(k_{1}, k_{2}, i\right)} ; \forall k_{1}, \forall k_{2}\right\}$ correspond to the $i^{\text {th }}$ task location ${ }^{w} \mathbf{T}_{\text {task }}^{(i)}$ and the indices $\left(k_{1}, k_{2}\right)$ are related to the sampled coordinates of the positioner and linear track respectively.

Using such presentation, the original continuous problem (1) is converted into a specific shortest-path problem on the graph, where all three successive nodes satisfy the acceleration constraints and the distances between two nodes $\mathbf{L}_{\text {task }}^{\left(k_{1}, k_{2}, i\right)}$ and $\mathbf{L}_{\text {task }}^{\left(k_{1}, k_{2}, i+1\right)}$ are equal to the technological tool displacement time from the $i^{\text {th }}$ to the $(i+1)^{\text {th }}$ task point, which is restricted by the maximum velocities and accelerations of the robot, the positioner and the linear track. It should be also noted that some of the nodes are excluded from the graph because of violation of the collision or singularity constraints as well as the joint limits. These nodes are marked as "inadmissible" ones in Figure 4, and they are not connected to any neighbour. So, the objective function to be minimized (robot motion time) 


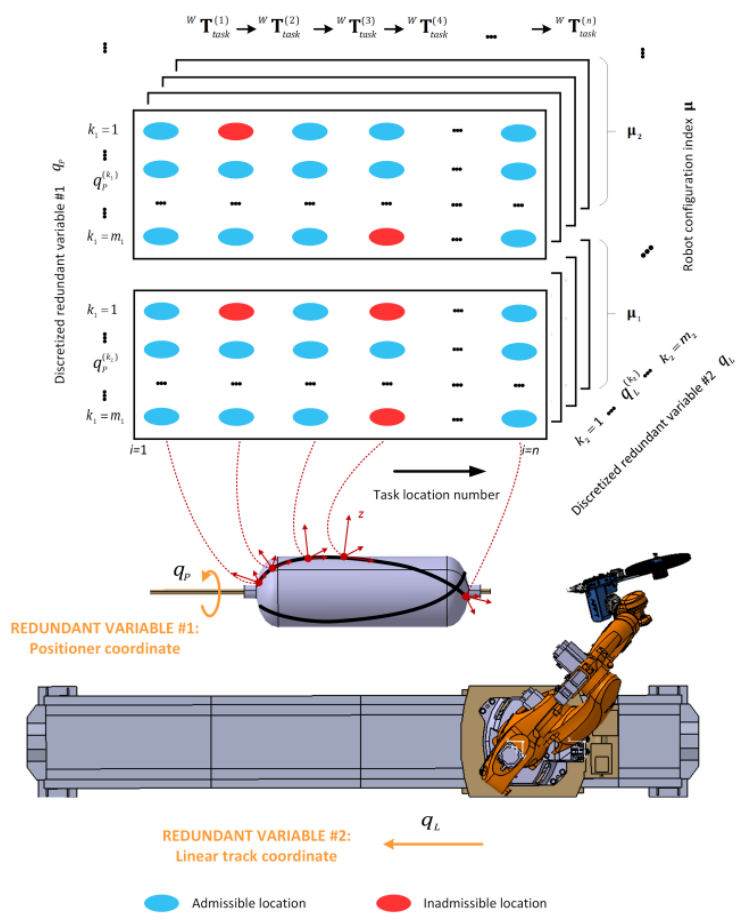

Figure 4: Task graph corresponding to the motion generation problem with two redundant variables.

can be presented as the sum of the edge weights

$$
T=\sum_{i=1}^{n-1} \operatorname{dist}\left(\mathbf{L}_{\text {task }}^{\left(k_{1}^{i}, k_{2}^{i}, i\right)}, \mathbf{L}_{\text {task }}^{\left(k_{1}^{i+1}, k_{2}^{i+1}, i+1\right)}\right) \rightarrow \min
$$

that is computed as

$$
\operatorname{dist}\left(\mathbf{L}_{\text {task }}^{\left(k_{1}^{i}, k_{2}^{i}, i\right)}, \mathbf{L}_{\text {task }}^{\left(k_{1}^{i+1}, k_{2}^{i+1}, i+1\right)}\right)=\max _{j=1,2, \ldots 8}\left(\frac{\left|q_{j}^{\left(k_{1}^{i}, k_{2}^{i}, i\right)}-q_{j}^{\left(k_{1}^{i+1}, k_{2}^{i+1}, i+1\right)}\right|}{\dot{q}_{j}^{\max }}\right)
$$

i.e. taking into account the maximum allowable velocities $\left\{\dot{q}_{j}^{\max } ; j=1,2, \ldots 8\right\}$ of the actuators. Corresponding optimal solution is represented by the sequence $\quad\left\{\mathbf{L}_{\text {task }}^{\left(k_{1}^{1}, k^{1}, 1\right)}\right\} \rightarrow\left\{\mathbf{L}_{\text {task }}^{\left(k_{1}^{2}, k_{2}^{2}, 2\right)}\right\} \rightarrow \ldots\left\{\mathbf{L}_{\text {task }}^{\left(k_{1}^{n}, n_{2}^{n}, n\right)}\right\} \quad$ that contains the actuated coordinates of the robot, positioner and linear track. It worth mentioning that the above expression straightforwardly takes into account the velocity constraints, while the acceleration constraints are verified by means of the second order approximation applied to the corresponding functions $\mathbf{q}_{R}(t), q_{P}(t)$ and $q_{L}(t)$ on the time interval $t \in\left[t_{i-1}, t_{i+1}\right]$. It allows us to present the acceleration constraints on the desired trajectory of the considered robotic system in the following form:

$$
\frac{2\left|\Delta t_{i} \Delta q_{j}^{(i+1)}-\Delta t_{i+1} \Delta q_{j}^{(i)}\right|}{\Delta t_{i+1} \Delta t_{i}\left(\Delta t_{i+1}+\Delta t_{i}\right)} \leq \ddot{q}_{j}^{\max }
$$

where $\Delta q_{j}^{(i)}=q_{j}^{\left(k_{1}^{i}, k_{2}^{i}, i\right)}-q_{j}^{\left(k_{1}^{i-1}, k_{2}^{i-1}, i-1\right)}$ and the time intervals $\Delta t_{i}$ are computed as the distance between the nodes $\mathbf{L}_{\text {task }}^{\left(k_{1}^{i}, k_{2}^{i}, i\right)}$ and $\mathbf{L}_{\text {task }}^{\left(k_{1}^{i-1}, k_{2}^{i-1}, i-1\right)}$.

To find the desired optimal path, conventional optimization techniques can be hardly applied because of extremely high computing time (Gao et al., 2017). Besides, these techniques are not able to take into account the acceleration constraints that are very essential here. For these reasons, a dedicated problem-oriented algorithm has been developed for this problem.

This algorithm is based on the dynamic programming principle, aiming at sequentially finding the shortest paths for the problems of lower dimensions, i.e. from $\left\{\mathbf{L}_{\text {task }}^{\left(k_{1}^{1}, k_{2}^{1}, 1\right)}, \forall k_{1}, \forall k_{2}\right\}$ to the current nodes $\left\{\mathbf{L}_{\text {task }}^{\left(k_{1}^{i}, k_{k}^{i}, i\right)}, \forall k_{1}, \forall k_{2}\right\}$. If the length of the corresponding shortest path is denoted as $d_{k_{1}, k_{2}, i}$, then the shortest path for the next locations $\left\{\mathbf{L}_{\text {task }}^{\left(k_{1}, k_{2}, i+1\right)}, \forall k_{1}, \forall k_{2}\right\}$ can be obtained by combining the optimal solutions for the previous column $\left\{\mathbf{L}_{\text {task }}^{\left(k_{1}^{\prime}, k^{\prime}, i\right)}, \forall k_{1}^{\prime}, \forall k_{2}^{\prime}\right\}$ and the distances between the task locations with the indices $i$ and $i+1$,

$$
d_{k_{1}, k_{2}, i+1}=\min _{k_{1}^{\prime}, k_{2}^{\prime}}\left\{d_{k_{1}^{\prime}, k_{2}^{\prime}, i}+\operatorname{dist}\left(\mathbf{L}_{\text {task }}^{\left(k_{1}, k_{2}, i+1\right)}, \mathbf{L}_{\text {task }}^{\left(k_{1}^{\prime}, k^{\prime}, i\right)}\right)\right\}
$$

This expression is applied recursively, starting from the second layer of the task graph $(i=2)$ and finishing by the last one $(i=n)$. So, the desired optimal path can be obtained after selection of the minimum length $d_{k_{1}, k_{2}, i+1}$ corresponding to the last layer. An outline of this path planning algorithm is presented in Appendix B. In fact, this algorithm is generalization of our previous technique that was developed for motion coordination of the robotic manipulator and positioner (without linear track). As follows from relevant study, this algorithm is rather time efficient in this more complicated case; which deals with two redundant axes.

\section{COMPARISON OF MOTION COORDINATION STRATEGIES}

To demonstrate advantages of the proposed technique, let us apply it to an industrial problem that deals with fabrication of a high-pressure composite vessel. Relevant robotic fiber placement workcell (see Figure 5) is composed of 6-axis serial robot KUKA KR210 R3100, 1-axis translational 
linear track KUKA KL2000 and 1-axis rotational positioner AFPT 550.

For comparison purposes, two cases will be considered where the robot base is assumed either fixed or movable by means of the linear track. These two cases correspond to different degrees of redundancy provided by the positioner only or by the positioner together with the linear track. For the first case, the technique described in our previous work (Gao et al., 2017) will be applied while the second case is based on the technique proposed in this paper.

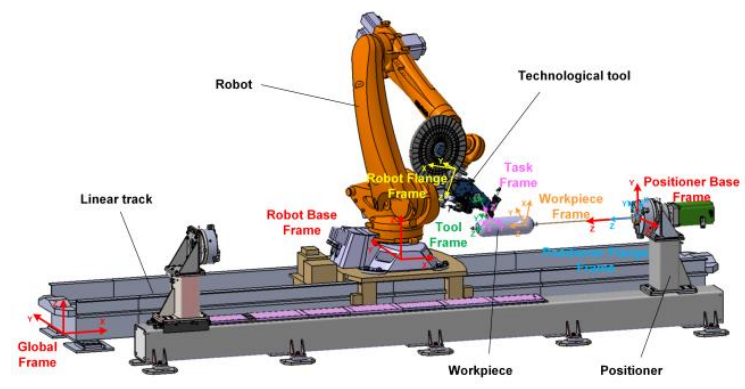

Figure 5: Robot-based fiber placement workcell and arrangement of coordinate frames.

\subsection{Optimal Motion Coordination for Fixed Robot Base}

A composite vessel considered in this case study is relatively small compared to the robot workspace. It is composed of a cylindrical part and two elliptical domes at both ends of the cylinder. The cylinder is $168 \mathrm{~mm}$ in diameter and $1200 \mathrm{~mm}$ in length. The laying task includes a single circuit placement of helical lamina. This allows executing the manufacturing task with fixed robot base, which simplifies the motion coordination but obviously leads to some increase of the total motion time. Nevertheless, here also arises another optimization problem that deals with optimal robot placement relative to the workpiece mounted on the positioner.

To find the optimal location of the robot base, the space of linear track coordinate (defining the robot placement) was sampled and the proposed motion planning technique was applied several times, assuming that the robot and the positioner coordination is required only. This yields the 2D task graph corresponding to a single redundant variable (positioner rotation angle), which was used to generate the time-optimal motions for each robot base location. Relevant results are presented in Table 1.
Table 1: Total motion time for different robot locations.

\begin{tabular}{|c|c|}
\hline Robot location [mm] & Motion time [sec] \\
\hline 2000 & 4.99 \\
\hline 2100 & 4.80 \\
\hline 2200 & 4.88 \\
\hline 2300 & $\mathbf{4 . 1 3}$ \\
\hline 2400 & 4.16 \\
\hline 2500 & 4.19 \\
\hline 2600 & 4.75 \\
\hline 2700 & 8.51 \\
\hline
\end{tabular}

As follows from the obtained results, the optimal robot location corresponds to the linear track coordinate $2300 \mathrm{~mm}$, which ensures the smallest motion time of $4.13 \mathrm{sec}$ to execute the desired technological task using capacities of the robot and the positioner only. It worth mentioning that this value is about $50 \%$ less compared to the non-optimal robot positioning when the desired path is located very close to the border of the robot workspace. In the following section, the obtained motion time will be compared with the similar value obtained for the case of movable robot base (when the actuation capacities of the linear track are also used).

\subsection{Optimal Motion Coordination for Movable Robot Base}

Another alternative for manufacturing the considered composite vessel is to actuate both positioner and linear track simultaneously, which corresponds to movable robot base. To coordinate motions of all mechanical components in this case, the joint coordinate spaces of both the positioner and the linear track were sampled with the step of $5^{\circ}$ and $15 \mathrm{~mm}$ respectively. Then, the proposed extension of the time-optimal motion generation technique that deals with two redundant variables was applied.

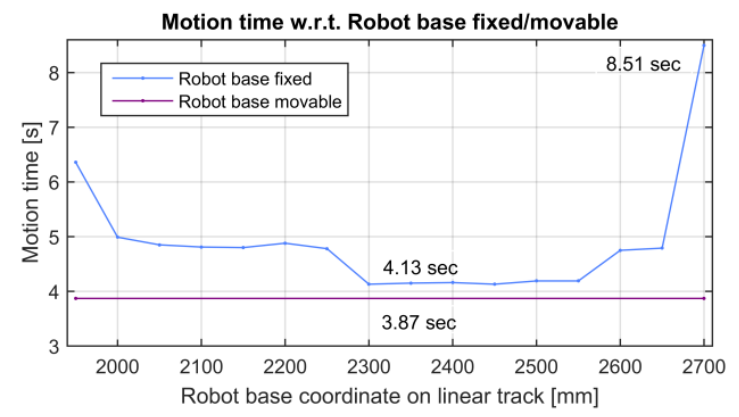

Figure 6: Total motion time for the cases of non-actuated and actuated linear track. 
Relevant computational results are presented in Figure 6. As follows from them, using movable robot base allows reducing the total motion time down to $3.87 \mathrm{sec}$, which is about $6 \%$ less compared to the case of the fixed robot base. The obtained trajectories are shown in Figures 7 and 8, which show the displacement and velocity profiles for all eight actuators of the 6 -axis robot, 1 -axis positioner and 1-axis linear track. It should be mentioned that after discrete optimization, a dedicated smoothing technique may be also used to locally improve the velocity and acceleration profiles and ameliorate the actuator working conditions.

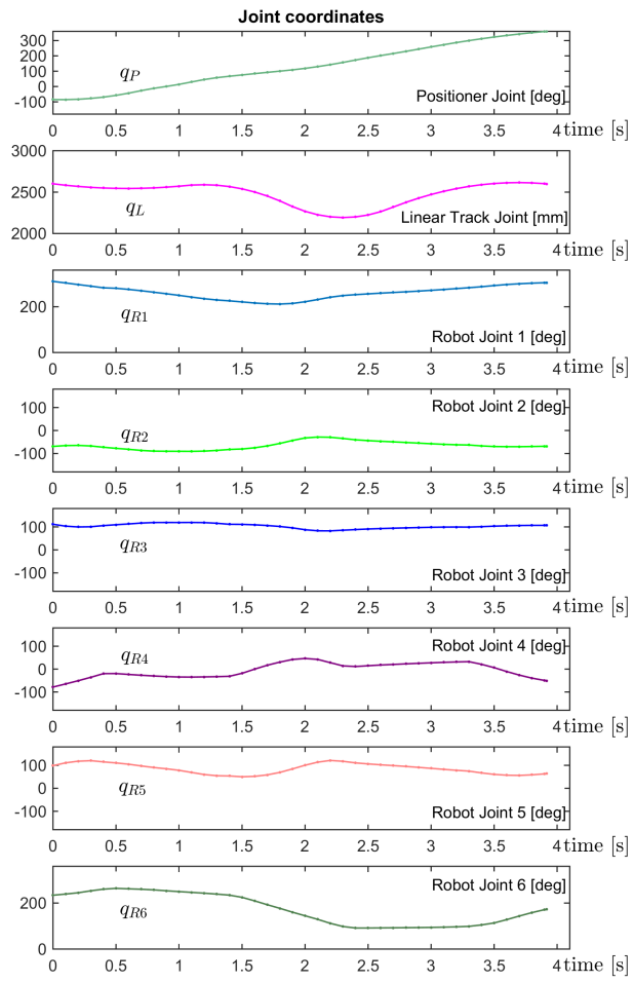

Figure 7: Displacement profiles of the coordinated timeoptimal motions of the robot, positioner and linear track.

\section{CONCLUSIONS}

This paper presents an extended technique dealing with the optimal motion coordination in redundant robotic fiber placement systems. In contrast to previous results dealing with a single redundant axis only, this technique allows the robot, positioner and linear track to be actuated simultaneously in order to reduce the total motion time. First, the original continuous optimization problem is converted into a combinatorial one where the desired timeoptimal motions are presented as a specific shortest path

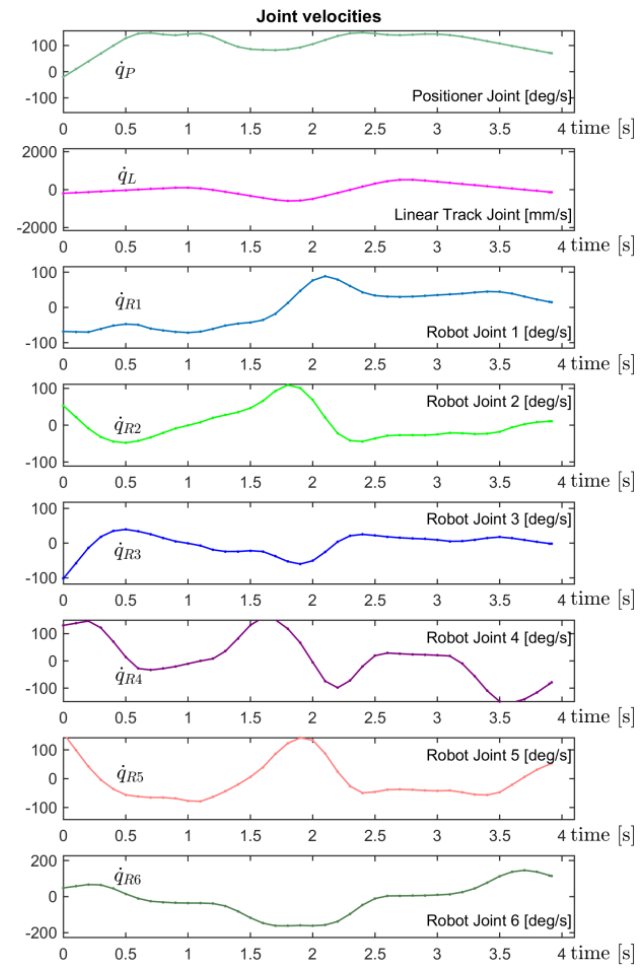

Figure 8: Velocity profiles of coordinated time-optimal motions of the robot, positioner and linear track.

on the task graph. Then, the desired time-optimal motions are generated using enhanced dynamic programming algorithm that takes into account the actuator capabilities (coordinate limits, velocities and accelerations) as well as the kinematic and geometric constraints allowing avoiding collisions and singular configurations of the manipulator. The proposed technique is illustrated by two case studies confirming simultaneous actuation of all robotic system axes. In future, the proposed technique will be generalized for the robotic systems with higher degrees of redundancy.

\section{ACKNOWLEDGEMENTS}

This work is supported by the China Scholarship Council (Grant $\mathrm{N}^{\circ} 210404490018$ ). The author also acknowledged CETIM for the motivation of this research work.

\section{REFERENCES}

Flacco, F. \& De Luca, A. 2015. Discrete-time redundancy resolution at the velocity level with acceleration/torque 
optimization properties. Robotics and Autonomous Systems, 70, 191-201.

Frketic, J., Dickens, T. \& Ramakrishnan, S. 2017. Automated manufacturing and processing of fiberreinforced polymer (FRP) composites: An additive review of contemporary and modern techniques for advanced materials manufacturing. Additive Manufacturing.

Gallet-Hamlyn, C. 2011. Multiple-use robots for composite part manufacturing. JEC composites, 28-30.

Gao, J., Pashkevich, A. \& Caro, S. 2017. Optimization of the robot and positioner motion in a redundant fiber placement workcell. Mechanism and Machine Theory, 114, 170-189.

Garoushi, S. 2018. Fiber-Reinforced Composites. In: MILETIC, V. (ed.) Dental Composite Materials for Direct Restorations. Cham: Springer International Publishing.

Gay, D. 2014. Composite materials: design and applications, CRC press.

Gueta, L. B., Chiba, R., Arai, T., Ueyama, T., Rubrico, J. I. U. \& Ota, J. 2017. Compact design of a redundant manipulator system and application to multiple-goal tasks with temporal constraint. Journal of Advanced Mechanical Design, Systems, and Manufacturing, 11, JAMDSM0012-JAMDSM0012.

Gueta, L. B., Chiba, R., Ota, J., Ueyama, T. \& Arai, T. Coordinated motion control of a robot arm and a positioning table with arrangement of multiple goals. Robotics and Automation, 2008. ICRA 2008. IEEE International Conference on, 2008. IEEE, 2252-2258.

Kazerounian, K. \& Nedungadi, A. 1988. Redundancy resolution of serial manipulators based on robot dynamics. Mechanism and machine theory, 23, 295303.

Pashkevich, A. P., Dolgui, A. B. \& Chumakov, O. A. 2004. Multiobjective optimization of robot motion for laser cutting applications. International Journal of Computer Integrated Manufacturing, 17, 171-183.

Pham, D.-C., Sridhar, N., Qian, X., Sobey, A. J., Achintha, M. \& Shenoi, A. 2016. A review on design, manufacture and mechanics of composite risers. Ocean Engineering, 112, 82-96.

\section{APPENDIX A TASK GRAPH GENERATION}

As mentioned in Section 4, here the pseudo-codes of the 3D task graph generation are presented. Its input includes is a sequence of $4 \times 4$ location matrices $\{\operatorname{Task}(i) \mid i=1,2, \ldots n\}$, the discretization densities $m_{1}$ and $m_{2}$, and the upper/lower limits of the redundant variables denoted as $q_{P}^{\text {max }}, q_{P}^{\min }$ and $q_{L}^{\text {max }}, q_{L}^{\min }$ respectively. The algorithm transforms the task locations Task(i) into the joint space.

This procedure contains two steps. Firstly, the redundant variables are uniformly discretized in the ranges of $\left[q_{P}^{\min }, q_{P}^{\max }\right]$ and $\left[q_{L}^{\min }, q_{L}^{\max }\right]$, and $m_{1} \times m_{2} \times n$ matrix $\left\{q_{P}\left(k_{1}, k_{2}, i\right), q_{L}\left(k_{1}, k_{2}, i\right)\right\} \quad$ is obtained, where $k_{1}=1,2, \ldots m_{1} ; k_{2}=1,2, \ldots m_{2}$ and $i=1,2, \ldots n$. Then, at the second step, $g_{P}(),. g_{L}($. and $g_{R}^{-1}($.) are sequentially applied, and the robot configuration states for $\left\{q_{P}\left(k_{1}, k_{2}, i\right), q_{L}\left(k_{1}, k_{2}, i\right) \mid \forall k_{1}, \forall k_{2}, \forall i\right\} \quad$ are computed. After checking with the joint limits, collision and the distance to singularities, the task graph is finally generated with nodes:

$$
\mathbf{L}\left(k_{1}, k_{2}, i\right)=\left\{q_{P}\left(k_{1}, k_{2}, i\right), q_{L}\left(k_{1}, k_{2}, i\right), \mathbf{q}_{R}\left(k_{1}, k_{2}, i\right)\right\}
$$

where $k_{1}=1,2, \ldots m_{1} ; k_{2}=1,2, \ldots m_{2}$ and $i=1,2, \ldots n$.

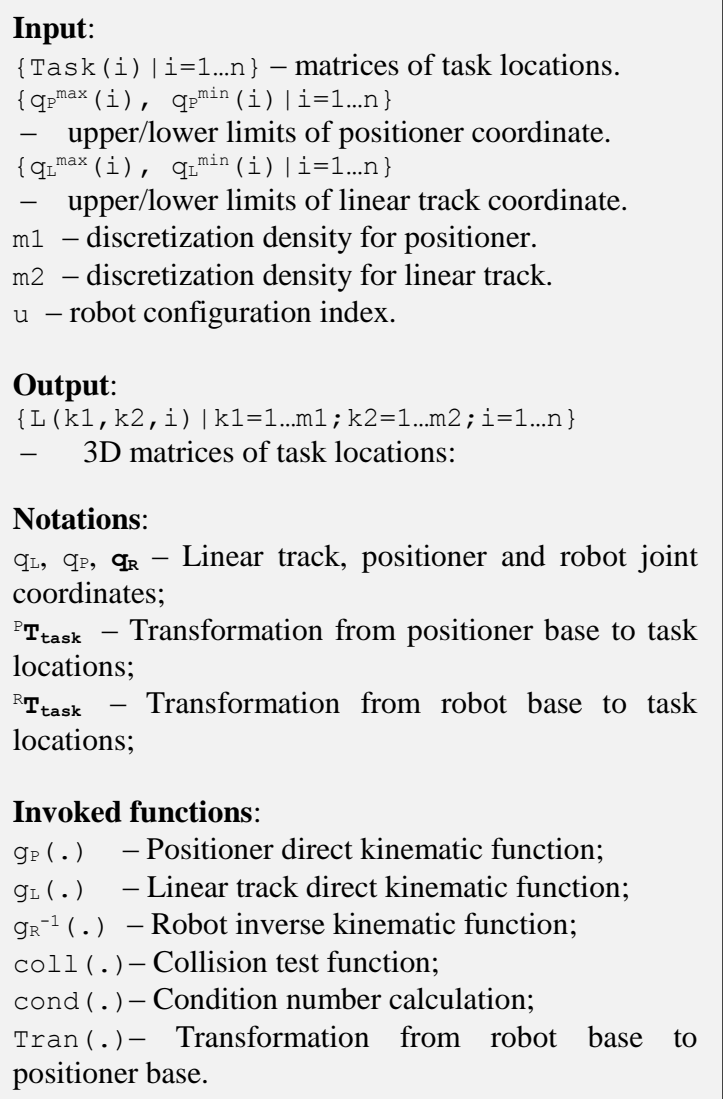

\section{Output:}

$\{\mathrm{L}(\mathrm{k} 1, \mathrm{k} 2, \mathrm{i}) \mid \mathrm{k} 1=1 \ldots \mathrm{m} 1 ; \mathrm{k} 2=1 \ldots \mathrm{m} 2 ; i=1 \ldots \mathrm{n}\}$

$q_{I}, q_{P}, q_{R}-$ Linear track, positioner and robot joint coordinates;

${ }^{\mathrm{P}} \mathbf{T}_{\text {task }}$ - Transformation from positioner base to task ocations

${ }^{\mathrm{R}} \mathbf{T}_{\text {task }}$ - Transformation from robot base to task Invoked functions:

$g_{\mathrm{P}}($.$) \quad - Positioner direct kinematic function;$

$g_{\mathrm{I}}($. ) - Linear track direct kinematic function,

$\mathrm{g}_{\mathrm{R}}^{-1}($. ) - Robot inverse kinematic function

cond (. ) - Condition number calculation;

positioner base. 


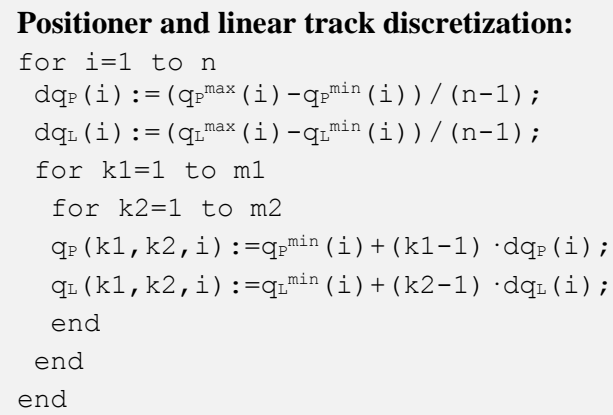

\section{Location matrix creation:}

for $i=1$ to $n$

\section{APPENDIX B SHORTEST PATH SEARCH}

Here, the pseudo-codes of the shortest path search are presented. The input is the $3 \mathrm{D}$ matrix of the locations $\left\{\mathbf{L}\left(k_{1}, k_{2}, i\right)\right\}$, which contains information on the configuration states satisfying the equality constraint, the collision constraint and the singularity constraint. The algorithm operates with two tables $D(k, i)$ and $P(k, i)$ that include the minimum distances for the sub-problem of lower size (for the path $1 \rightarrow i$ ) and the pointers to the previous locations respectively.

The procedure is composed of four basic steps. The step (1) reshapes the 3D graph to the one with $m_{1} \times m_{2}$ lines and $n$ columns for simplifying the programming. In step (2), the recursive formula (6) is implemented. For the admissible configuration nodes, the acceleration constraints are examined using the expression (5) for each candidate path connecting the nodes with the indices $i, i-1$ and $i-2$. It is worth mentioning that the function $\operatorname{accl}(\cdot)$ requires three inputs corresponding to $i, i-1$ and $i-2$, but the location for $i-2$ is determined using the pointer $P(j, i-1)$ to the previous location in the current path. Then, it finds the minimum path from the current node to the first column and records the reference into the pointer matrix. In steps (3) and (4), the optimal solution is finally obtained and corresponding path is extracted by means of the backtracking.

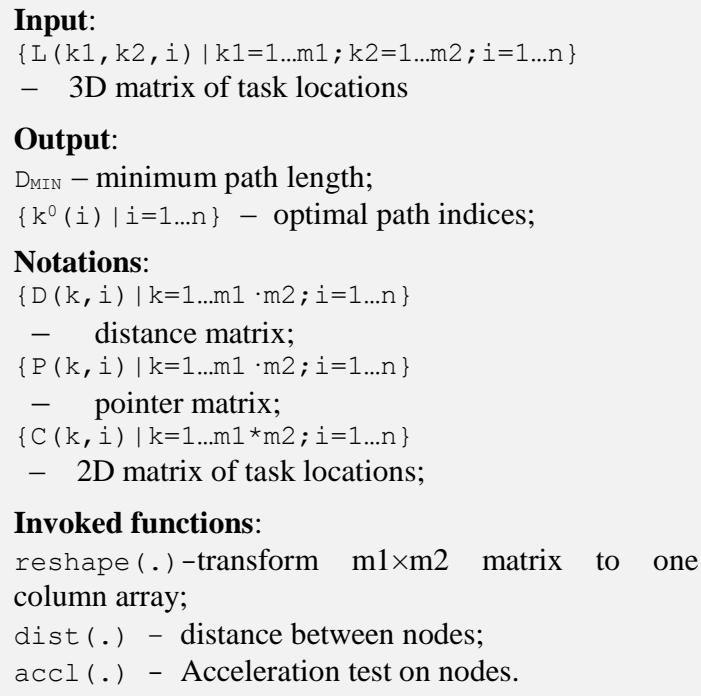

\section{Invoked functions:}

reshape(.)-transform $\mathrm{m} 1 \times \mathrm{m} 2$ matrix to one column array;

dist (.) - distance between nodes;

$\operatorname{accl}()-$. Acceleration test on nodes.

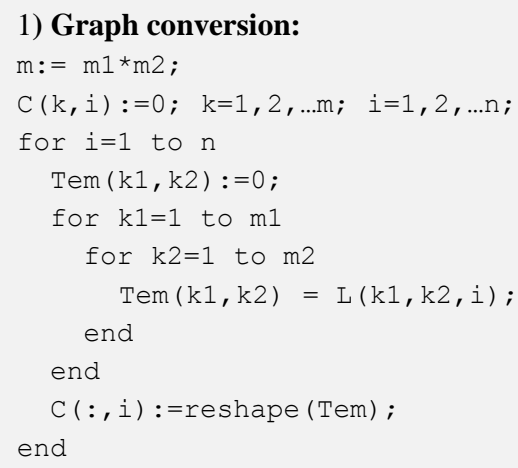

\section{3) Shortest path selection:}

set $\operatorname{DMIN}_{\text {Min }}:=\min (\{\mathrm{D}(\mathrm{k}, \mathrm{n}) \mid \mathrm{k}=1,2, \ldots \mathrm{m}\})$; $k^{0}(n):=\operatorname{argmin}(\{r(j) \mid j=1,2 \ldots m\})$;

\section{4) Backtracking:}

for $i=n$ to 2

$k^{0}(i-1):=P\left(k^{0}(i), i\right)$;

end 
The above presented algorithm has been tested using Matlab 2016b environment (running at Intel ${ }^{\circledR} \mathrm{i} 5 \mathrm{CPU}$ @ $2.67 \mathrm{GHz} 2.67 \mathrm{GHz}$ ). In the case of the fixed robot base, it took about one minute to generate the timeoptimal motions for each sampled robot location. In the case of the movable robot base, the computation required about one hour. It is clear that in other programming environment, the computing time would be essentially smaller. 